\title{
Analytical Model for the Assessment of Efficiency of Stem Cell Transplantation with Suicidal Gene Construct for the Treatment of Leukemia
}

\author{
Probir Kumar Dhar ${ }^{1,2,3}$, Tarun Kanti Naskar ${ }^{1,3}$ and Durjoy Majumder ${ }^{1,4 *}$ \\ ${ }^{1}$ Society for Systems Biology \& Translational Research, 103, Block - C, Bangur Avenue, Kolkata 700 055, India \\ ${ }^{2}$ Department of Electronics and Communication Engineering, Bengal College of Engineering \& Technology, Sahid Sukumar Banerjee Sarani, Bidhannagar, Durgapur \\ 713212, Burdwan, West Bengal, India \\ ${ }^{3}$ Department of Mechanical Engineering, Jadavpur University, 188 Raja S. C. Mallick Road, Kolkata 700032, India \\ ${ }^{4}$ Department of Physiology, West Bengal State University, Berunanpukuria, Malikapur, Barasat, Kolkata 700126, India
}

\begin{abstract}
Hematopoietic stem cell transplantation is now being the emergent methodology for leukemia treatment. Due to HLA mis-match, there is chance of transplantation related mortality. However, with the increase in HLA mis-match between donor cells and recipient, there is more chance of complete removal of leukemic cells in host (patient). To tackle this, recently stem cells transplantation with suicidal $t k$-gene construct is being suggested. Due to unavailability of suitable analytical methods this option has limited applications in clinical cases. Present work provides an analytical platform to test the efficacy of this therapeutic procedure.
\end{abstract}

Keywords: Difference delay equation; Leukemia; HLA; Hematopoietic stem cell; $t k$ gene

\section{Introduction}

Hematopoietic stem cell transplantation (HSCT) is considered to be the emerging therapeutic procedure for leukemia treatment. And hence a lot of clinical trials have been done across the globe. Generally allogenic transplantation is being made and the success rate depends on HLA (Human Leukocytic Antigen) incompatibility between the donor and recipient. The degree of HLA incompatibility enhances the killing efficiency of leukemic cell(s); contrarily it decreases the quality of life of the concerned patient due to enhanced GVHD (Graft Versus Host Disease). Therefore stem cell transplantation is being regarded as the 'double edge sword' [1,2]. Hence HSCT requires a judicious choice of donor cell selection. Though there is availability of rich medical and clinical literature on HSCT; however, most of the literatures are based on empirical observations and physicians personal experiences. This is due to unavailability of proper analytical method for such therapeutic procedure.

Though several analytical models are available for leukemia pathogenesis and dynamics, imatinib treatment, myeloablative chemotherapy, immune potentiation effect for different types of leukemia (CML, Chronic Myelogenous Leukemia; AML, Acute Myeloblastic Leukemia); but no single model was available to test the efficacy of different combinations of therapy for any particular leukemia type for any particular individual patient [3-15]. Recent time different therapeutic strategies along with their combinations can be assessed for different types of leukemia with a single analytical model [16]. Through this model the efficacy of myeloablative and low dose chemotherapy along with supportive therapy like RBC and platelet transfusion, cytokine therapy, imatinib therapy, allogenic HSCT with different degree of HLA matching, effect of GVHD and immunosuppressive drug after HSCT can be tested.

In order to optimize the situation i.e., to enhance malignancy removal together with improved safety of allogenic transplantation, recently allogenic HSCT having HLA incompatibility with a $t k$ (thymidine kinase) gene construct has been suggested. This therapeutic procedure has two way advantages - one, complete removal of leukemic cells (Graft Versus Leukemia reaction, GVL) due to high HLA mismatch and the other, $t k$ gene construct provide the advantage of controlling GVHD by selective killing of transplanted cells with the application of pro-drug Gangcyclovir $[17,18]$. Though it seems to be safer, however such therapeutic procedure is a risky one as with the delay in application of pro-drug gangcyclovir, there is a high chance of transplanted related mortality. A suitable analytical method is required that can predict the minimal residual disease (MRD) and the optimal timing for application of pro-drug.

\section{Analytical Model}

\section{Model description}

In the previous work [16], different therapies namely myeloablative chemotherapy (myl), cytokine (CYT), RBC $\left(T_{R B C}\right)$ and platelet $\left(T_{\text {PLATELET }}\right)$ transfusion (transfuse in subscript or $t f$ ), allogenic HSCT $\left(S_{e}\right)$, immuo-suppressive drug $(\mathrm{im})$ in case of HLA incompatibility are being operative through following equation.

$$
\begin{aligned}
& x(k)=A x(k-1)+B^{1} x\left(k-d k_{1}\right)+B^{2} x\left(k-d k_{2}\right)+B^{3} x\left(k-d k_{3}\right)+B^{4} x\left(k-d k_{4}\right)+ \\
& R B C_{\text {transfuse }} T_{R B C}(k)+P L A T E L E T_{\text {transfuse }} T_{\text {PLATELET }}(k) \\
& \text { or, }\left[x_{1}(k) ; x_{2}(k) ; \ldots ; x_{28}(k)\right]=\left[a_{1,1}, \ldots, a_{1,28} ; \ldots ; a_{28,1}, \ldots, a_{28,28}\right] \times\left[x_{1}(k-\right.
\end{aligned}
$$

*Corresponding author: Durjoy Majumder, Department of Physiology, West Bengal State University, Berunanpukuria, Malikapur, Barasat, Kolkata 700 126, India, Tel: 033 25241979; E-mail: durjoy@rocketmail.com or durjoy@ssbtr.net

Received September 28, 2015; Accepted November 26, 2015; Published December 02, 2015

Citation: Dhar PK, Naskar TK, Majumder D (2015) Analytical Model for the Assessment of Efficiency of Stem Cell Transplantation with Suicidal Gene Construct for the Treatment of Leukemia. J Oncol TransI Res 1: 103. 10.4172/2476-2261.1000103

Copyright: @ 2015 Dhar PK, et al. This is an open-access article distributed under the terms of the Creative Commons Attribution License, which permits unrestricted use distribution, and reproduction in any medium, provided the original author and source are credited. 
Citation: Dhar PK, Naskar TK, Majumder D (2015) Analytical Model for the Assessment of Efficiency of Stem Cell Transplantation with Suicidal Gene Construct for the Treatment of Leukemia. J Oncol Transl Res 1: 103. 10.4172/2476-2261.1000103

$\left.x_{2}\left(k-d k_{3}\right) ; \ldots ; x_{28}\left(k-d k_{3}\right)\right]+\left[b^{4}{ }_{1,1}, \ldots, b^{4}{ }_{1,28} ; \ldots ; b_{28,1}^{4}, \ldots, b^{4}{ }_{28,28}\right] \times\left[x_{1}(k-\right.$ $\left.\left.d k_{4}\right) ; x_{2}\left(k-d k_{4}\right) ; \ldots ; x_{28}\left(k-d k_{4}\right)\right]+\left[R B C t f_{1,1} ; R B C t f_{2,1} ; \ldots ; R B C t f_{28,1}^{28,2}\right] \times T_{R B C}(k)$ $+\left[\right.$ PLATELETtf $f_{1,1} ;$ PLATELETtf $f_{2,1} ; \ldots ;$ PLATELETtf $\left.{ }_{28,1}\right] \times T_{\text {PLATELET }}(k)$,

where the elements of matrix A $_{\text {are }} a_{1,1}(k)=\left(1+m_{S g}-a_{s g}-S g d r-C S_{g s}-C S_{g r}\right.$ $-C_{m y l} \times M y l_{s g} \times d_{m y l}(k)-C_{i m} \times$ imsens $\left._{S g} \times d_{i m}(k)\right), a_{2,2}(k)=\left(1+m_{s s}-a_{S s}-S s d r-C S_{s g}^{g s}\right.$ $C S_{s r}-C_{m y l} \times M y l_{s s} \times d_{m y l}(k)-C_{i m} \times$ imsens $\left._{S s} \times d_{i m}(k)\right), a_{33}(k)=\left(1+m_{s r}-a_{s r}-S r d r-\right.$ $C S_{r s}-C S_{r g}-C_{m y l} \times M y l_{S r} \times d_{m y l}(k) \quad-C_{i m} \times$ imsens $\left._{S r} \times d_{i m}(k)\right), \quad a_{4,4}(k)=\left(1+m_{P I g}-\right.$ $a_{P I g}-P 1 g d r-C P 1_{g s}-C P 1_{g r} \quad-C_{m y l} \times M y l_{P I g} \times d_{m y l}(k)-C_{i m} \times$ imsens $\left._{P 1 g} \times d_{i m}(k)\right)$,

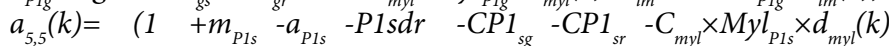
$-C_{i m} \times$ imsens $\left._{P 1 s} \times d_{i m}(k)\right), \quad a_{6,6}(k)=\left(1+m_{P 1 r}-a_{P 1 r}-P 1 r d r-C P 1_{r g}-C P 1_{r s}\right.$ $-C_{m y l} \times M y l_{P 1 r} \times d_{m y l}(k)-C_{i m} \times$ imsens $\left._{P 1 r} \times d_{i m}(k)\right), \quad a_{7,7}(k)=\left(1+m_{P 2 g}-a_{P 2 g}\right.$ $P 2 g d r-C P 2_{g s}-C P 2_{g r}-C_{m y l} \times M y l_{P 2 g} \times d_{m y l}(k)-C_{i m} \times$ imsens $_{P 2 g} \quad \times d_{i m}(k)$ $\left.+C_{C Y T} \times d_{C Y T}^{g^{s}}(k) \times m_{C Y T}\right), \quad a_{8,8}(k)=\left(\begin{array}{llll}1+m_{P 2 s}-a_{P 2 s} & -P 2 s d r & -C P 2_{s g} & -C P 2_{s r}\end{array}\right.$ $-C_{m y l} \times M y l_{P 2 s} \times d_{m y l}(k) \quad-C_{i m} \times$ imsens $\left._{P 2 s} \times d_{i m}(k)\right), \quad a_{9,9}(k)=\left(1+m_{P 2 r}-a_{P 2 r}-\right.$ $P 2 r d r-C P 2_{r g}-C P 2_{r s}-C_{m y l} \times M y l_{P 2 r} \quad \times d_{m y l}(k)-C_{i m} \times$ imsens $\left._{P 2 r} \times d_{i m}(k)\right)$, $a_{10,10}(k)=\quad\left(1+m_{P 3 g}-a_{P 3 g}-P 3 g d r-C P 3_{g s} \quad-C P 3_{g r}-C_{m y l} \times M y l_{P 3 g} \times d_{m y l}(k)-\right.$ $C_{i m} \times$ imsens $\left._{P 3 g} \times d_{i m}(k)\right), \quad a_{11,11}(k)=\left(1+m_{P 3 s}-a_{P 3 s}-P 3 s d r-C P 3_{s g}-C P 3_{s r}\right.$ $-C_{m y l} \times M y l_{P 3 s} \times d_{m y l}(k)-C_{i m} \times$ imsens $\left._{P 3 s} \times d_{i m}(k)\right), a_{12,12}(k)=\left(1+m_{P 3 r}-a_{P 3 r}\right.$ $-P 3 r d r \quad-C P 3_{r g}-C P 3_{r s}-C_{m y l} \times M y l_{P 3 r} \times d_{m y l}(k)-C_{i m} \times$ imsens $\left._{P 3 r} \times d_{i m}(k)\right)$, $a_{13,13}(k)=\left(1-a_{B 1 g}-C B 1_{g s}-C B 1_{g r}-C_{m y l} \times M y l_{B 1 g} \times d_{m y l}(k)-C_{i m} \times i^{2 m s e n s} s_{B 1 g}\right.$ $\left.\times d_{i m}(k)\right), \quad a_{14,14}(k)=\left(1-a_{B 1 s}-C B 1_{s g}-C B 1_{s r} \quad-C_{m y l} \times M y l_{B 1 s} \times d_{m y l}(k)\right.$ $-C_{i m} \times$ imsens $\left._{B 1 s} \times d_{i m}(k)\right), \quad a_{15,15}(k)={ }^{B g}\left(1-a_{B 1 r}-C B 1_{r g}-C B 1_{r s}-C_{m y l}\right.$ $\left.\times M y l_{B 1 r} \times d_{m y l}(k)-C_{i m} \times i^{2 m s e n s} s_{B 1 r} \times d_{i m}(k)\right), \quad a_{16,16}(k)=\left(1-a_{B 2 g}-C B 2_{g s}-C B 2_{g r}\right.$ $-C_{m y l} \times M y l_{B 2 g} \times d_{m y l}(k)-C_{i m} \times$ imsens $\left._{B 2 g} \times d_{i m}(k)\right), a_{17,17}(k)=\left(1-a_{B 2 s}-C B 2_{s g}^{g r}\right.$ $\left.-C B 2_{s r}-C_{m y l} \times M y l_{B 2 s} \times d_{m y l}(k)-C_{i m} \times i^{2} \operatorname{sens} s_{B 2 s} \times d_{i m}(k)\right), a_{18,18}(k)=\left(1-a_{B 2 r}\right.$ $\left.-C B 2_{r g}-C B 2_{r s}-C_{m y l} \times M y l_{B 2 r} \times d_{m y l}(k)-C_{i m} \times i m s e n s_{B 2 r} \times d_{i m}(k)\right), \quad a_{19,19}(k)$

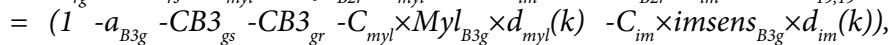

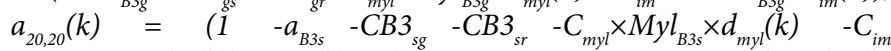
$\times$ imsens $\left._{B 3 s} \times d_{i m}(k)\right), a_{21,21}(k)=\left(1-a_{B 3 r}-C B 3_{r g}-C B 3_{r s}-C_{m y l} \times M y l_{B 3 r} \times d_{m y l}(k)\right.$ $-C_{i m}$ ximsens $\left._{B 3 r} \times d_{i m}(k)\right), \quad a_{22,22}(k)=\left(1+m_{S e}-a_{S e}-S e d r-C_{i m} \times\right.$ imsens $_{S e}$ $\left.\times d_{i m}(k)\right), \quad a_{23,23}(k)=\left(1+m_{P 1 e}-a_{P 1 e}-P 1 e d r-C_{i m} \times\right.$ imsens $\left._{P 1 e} \times d_{i m}(k)\right), \quad a_{24,24}(k)$ $=\left(1+m_{P 2 e}-a_{P 2 e}-P 2 e d r-C_{i m} \times\right.$ imsens $\left._{P 2 e} \times d_{i m}(k)+C_{C Y T} \times d_{C Y T}(k) \times m_{D C Y T}\right)$, $a_{25,25}(k)=\left(1+m_{P 3 e}-a_{P 3 e}-P 3 e d r-C_{i m} \times\right.$ imsens $\left._{P 3 e} \times d_{i m}(k)\right), \quad a_{26,26}(k)=(1$ $\left.-a_{B 1 e}-C_{i m} \times i^{25 m e n s} s_{B 1 e} \times d_{i m}(k)\right), \quad a_{27,27}(k)=\left(1-a_{B 2 e}-C_{i m} \times i^{i m s e n s} s_{B 2 e} \times d_{i m}(k)\right)$, $a_{28,28}(k)=\left(1-a_{B 3 e}-C_{i m}^{B 1 e} \times\right.$ imsens $\left._{B 3 e} \times d_{i m}^{27,27}(k)\right)$. Multiplication rate, apoptosis rate and differentiation rate of concerned cell type are denoted by $m, a$ and $d r$ respectively.

Due to mutability one category cell is converted to another one, say normal $(g)$ to resistant $(r)$ or sensitive $(s)$ category of different types of cells [stem cell $(S)$, erythroblast $(P 1)$, lymphoblast $(P 2)$, megakaryoblast $(P 3), \mathrm{RBC}(B 1), \mathrm{WBC}(B 2)$, platelet $(B 3)]$. The conversion rates $(C)$ from one category (represented by first subscript) to another (represented by second subscript) are represented by the following matrix elements.

$a_{1,2}=C S_{s g^{\prime}} \quad a_{1,3}=C S_{r g}, a_{2,1}=C S_{g s}, a_{2,3}=C S_{r s}, a_{3,1}=C S_{g r}, a_{3,2}=C S_{s r^{\prime}} \quad a_{4,5}$ $=C P 1_{s g^{\prime}} a_{4,6} \stackrel{s g^{\prime}}{=} C P 1_{r g}, a_{5,4}=C P 1_{g s}, a_{5,6}=C P 1_{r s}, a_{6,4}=C P 1_{g r}, a_{6,5}=C P 1_{s r^{\prime}} a_{7,8}=$ $C P 2_{s g^{\prime}} a_{7,9}=C P 2_{r g}, a_{8,7}=C P 2_{g s}, a_{8,9}=C P 2_{r s}, a_{9,7}=C P 2_{g r}, a_{9,8}=C P 2_{s r}, a_{10,11}=$

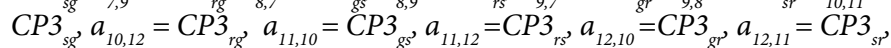
$a_{13,14}=C B 1_{s g}, a_{13,15}=C B 1_{r g}, a_{14,13}=C B 1_{g s}, a_{14,15}=C B 1_{r s}, a_{15,13}=C B 1_{g r}, a_{15,14}=$ $C B 1_{s r^{\prime}} a_{16,17}=C B 2_{s g^{\prime}} a_{16,18}=C B 2_{r g^{\prime}} a_{17,16}=C B 2_{g g^{\prime}} a_{17,18}=C B 2_{r r^{\prime}} a_{18,16}=C B 2_{g r^{\prime}}$ $a_{18,17}=C B 2_{s r^{\prime}} a_{19,20}=C B 3_{s g^{\prime}} a_{19,21}=C B 3_{r g^{\prime}} a_{20,19}=C B 3_{g s}, a_{20,21}=C B 3_{r s^{\prime}} a_{21,19}$ $=C B 3_{g r}, a_{21,20}=C B 3_{s r}$. In case of simulation study conversion rates were assumed to be zero.

Following matrix elements are representing the immunity related killing of malignant cells and are time varying functions depending on host lymphocyte $(B 2 g)$ cell count.

$a_{2,16}(k)=-B 2 g \_S s(k), \quad a_{3,16}(k)=-B 2 g \_S r(k), \quad a_{8,16}(k)=-B 2 g \_P 2 s(k)$, $a_{9,16}(k)=-B 2 g_{-} P 2 r(k), a_{17,16}(k)=-B 2 g_{-} B 2 s(k)+C B 2_{g s}, a_{18,16}(k)=-B 2 g_{-}$ $B 2 r(k)+C B 2_{g r}$
Following matrix elements are changed due to malignancy related toxicity development [16].

$$
\begin{aligned}
& a_{1,2}=-0.001+C S_{s g^{\prime}} a_{1,3}=-0.001+C S_{r g^{\prime}} a_{4,5}=-1+C P 1_{s g^{\prime}} a_{4,6}=-1+C P 1_{r g^{\prime}} \\
& a_{10,11}=-1+C P 3_{s g^{\prime}} a_{10,12}=-1+C P 3_{r g^{\prime}} a_{22,2}=-0.001, a_{22,3}=-0.001, a_{23,5}=-1, \\
& a_{23,6}=-1, a_{25,11}=-1, a_{25,12}=-1 .
\end{aligned}
$$

Following matrix elements are changed due to cachexia related killing of host cells and exogenous transplanted $(T)$ cells ( $T=1$, after transplantation, otherwise $T=0$ ) by $P 2 r$ development.

$a_{1,9}=-0.000011, a_{4,9}=-0.000825, a_{7,9}=-0.000825+C P 2_{r g}, a_{10,9}=$ $-0.000825, a_{13,9}=-0.00275, a_{16,9}=-0.000275, a_{19,9}=-0.000275, a_{22,9}=$ $-T \times 0.000011, a_{23,9}=-T \times 0.000825, a_{24,9}=-T \times 0.000825, a_{25,9}=-T \times 0.000825$, $a_{26,9}=-T \times 0.000011, a_{27,9}=-T \times 0.000011, a_{28,9}=-T \times 0.000011$.

Similarly, following matrix elements are changed due to cachexia related killing by $P 2 s$ development.

$a_{1,8}=-0.000006, a_{4,8}=-0.00045, a_{7,8}=-0.00045+C P 2_{s g^{\prime}} a_{10,8}=-0.00045$, $a_{13,8}=-0.0015, a_{16,8}=-0.0015, a_{19,8}=-0.0015, a_{22,8} \stackrel{s g}{=} T \times 0.000006$, $a_{23,8}=-T \times 0.00045, a_{24,8}=-T \times 0.00045, a_{25,8}=-T \times 0.00045, a_{26,8}=-T \times 0.0015$, $a_{27,8}=-T \times 0.0015, a_{28,8}=-T \times 0.0015$.

Similarly, following matrix elements are changed due to cachexia related killing by $B 2 r$ development.

$a_{1,18}=-0.000003, a_{4,18}=-0.0003375, a_{7,18}=-0.0003375, a_{10,18}=$ $-0.0003375, a_{13,18}=-0.00075, a_{16,18}=-0.00075+C B 2_{r g^{\prime}} a_{19,18}=-0.00075$, $a_{22,18}=-T \times 0.000003, a_{23,18}=-T \times 0.0003375, a_{24,18}=-T \times 0.0003375$, $a_{25,18}=-T \times 0.0003375, a_{26,18}=-T \times 0.00075, a_{27,18}=-T \times 0.00075, a_{28,18}=-T \times$ 0.00075 .

Likewise, following matrix elements are changed due to cachexia related killing by $B 2 s$ development.

$a_{1,17}=-0.000003, a_{4,17}=-0.0003375, \quad a_{7,17}=-0.0003375, a_{10,17}=$ $-0.0003375, a_{13,17}=-0.00075, a_{16,17}=-0.00075+C B 2_{s g}, a_{19,17}=-0.00075$, $a_{22,17}=-T \times 0.000003, \quad a_{23,17}=-T \times 0.0003375, \quad a_{24,17}=-T \times 0.0003375$, $a_{25,17}=-T \times 0.0003375, \quad a_{26,17}=-T \times 0.00075, a_{27,17}=-T \times 0.00075, a_{28,17}=$ $-T \times 0.00075$.

Following matrix elements are changed due to GVHD related killing of donor cell by host lymphocytes.

$a_{22.16}=-T \times G \times s t \_m u l \times k i l l{ }_{b 2 g \rightarrow D}, a_{23,16}=-T \times G \times p r o \_m u l \times e f f e c t$ ple $\times$ kill ${ }_{b 2 g \rightarrow D}, a_{24,16}=-T \times G \times$ pro_mul $\times$ effect_p2e $\times k_{i l l} l_{b 2 g \rightarrow D}, a_{25,16}=-T \times G$ $\times p r o \_m u l \times e f f e c t \_p 3 e \times k i l l_{b 2 g \rightarrow D}, a_{26,16}=-T \times G \times$ mat_mul $\times k i l l b_{b 2 \Omega \rightarrow R B C}, a_{27,16}$ $=-T \times G \times$ mat_mul $\times k i l l_{b 2 g \rightarrow D}, a_{28,16}=-T \times G \times$ mat_mul $\times k i l l_{b 2 g \rightarrow D}$. The degree of HLA mis-match is represented through $G$.

Following matrix elements are changed due to GVHD related killing of host normal cells by donor lymphocytes. $a_{1,27}(k)=-T \times G \times s t_{-} m u l \times k i l l_{b 2 e \rightarrow g}, a_{4,27}(k)=-T \times G \times p r o \_m u l \times k i l l_{b 2 e \rightarrow g} \times$ $G V H D \_k i l l \_p 1 g(k-1), a_{7,27}(k) \stackrel{b 2 e \rightarrow g}{=}-T \times G \times p r o \_m u l \times k i l l{ }_{b 2 e \rightarrow g} \times G V H D \_k i l l$ $p 2 g(k-1), a_{10,27}(k)=-T \times G \times p r o \_m u l \times k i l l l_{b 2 e \rightarrow g} \times G V H D \_k i l l \_p 3 g(k-1)$, $a_{13,27}=-T \times G \times$ mat_mul $\times k_{i l l} l_{b 2 e \rightarrow R B C}, \quad a_{16,27}=-T \times G \times$ mat_mul $\times k i l l_{b 2 e \rightarrow g^{\prime}}$ $a_{19,27}=-T \times G \times$ mat_mul $\times k_{i l l} l_{b 2 e \rightarrow g}$.

Following matrix elements are changed due to GVL related killing of malignant cells by donor lymphocytes.

$a_{2,27}=-T \times s t \_m u l \times k i l l_{b 2 e \rightarrow h m}, \quad a_{3,27}=-T \times s t \_m u l \times k i l l_{b 2 e \rightarrow h m}, \quad a_{5,27}=$ -T×pro_mul $\times k i l l l_{b 2 e \rightarrow h m}, a_{6,27}=-T \times p r o \_m u l \times k i l l l_{b 2 e \rightarrow h m}, a_{8,27}=-T \times$ pro_mul $\times k_{i l l}{ }_{b 2 e \rightarrow h m}, a_{9,27}=-T \times p r o \_m u l \times k i l l l_{b 2 e \rightarrow h m}, a_{11,27}=-T \times$ pro_mul $\times k i l l l_{b 2 e \rightarrow h m}$, $a_{12,27} \stackrel{b 2 \rightarrow h}{=} \quad \times$ pro_mul $\times$ kill $_{b 2 e \rightarrow h m}, \quad a_{14,27}=-T \times m a t \_m u l \times k i l l b_{b 2 e \rightarrow h m^{\prime}}$ $a_{15,27}=-T \times m a t \_m u l \times k i l l_{b 2 e \rightarrow h m}, \quad a_{17,27}=-T \times$ mat_mul $\times k i l l_{b 2 e \rightarrow h m}, \quad a_{18,27}$ 
Citation: Dhar PK, Naskar TK, Majumder D (2015) Analytical Model for the Assessment of Efficiency of Stem Cell Transplantation with Suicidal Gene Construct for the Treatment of Leukemia. J Oncol Transl Res 1: 103. 10.4172/2476-2261.1000103

$=-T \times m a t \_m u l \times k i l l_{b 2 e \rightarrow h m}, a_{20,27}=-T \times m a t \_m u l \times k i l l_{b 2 e \rightarrow h m}, a_{21,27}=-T \times m a t_{-}$ mul $\times k i l l{ }_{22 c \rightarrow m}$. The parametric values of st_mul, pro_mul, mat_mul,

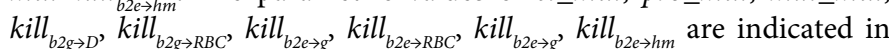
[16]. The rest of the elements of matrix $A$ are zero.

The matrix elements of $B^{1}, B^{2}, B^{3}, B^{4}, R B C_{\text {transfuse }}$ and PLATELET $T_{\text {transfuse }}$ matrixes are as follows.

$b_{4,1}^{1}=b_{7,1}^{1}=b^{1}{ }_{10,1}=\operatorname{Sgdr} / 3, b_{5,2}^{1}=b_{8,2}^{1}=b_{11,2}^{1}=\operatorname{Ssdr} / 3, b_{6,3}^{1}=b_{9,3}^{1}=b^{1}{ }_{12,3}$ $=S r d r / 3, b^{2}{ }_{134}=P 1 g d r, b^{2}{ }_{14,5}=P 1 s d r, b^{2}{ }_{15,6}=P 1 r d r, b_{26,23}^{2}=P 1 e d r, b^{3}{ }_{16,7}=$ $P 2 g d r, b_{17,8}^{3}=P 2 s d r, b_{18,9}^{3}=P 2 r d r, b_{27,24}^{3}=P 2 e d r, b_{19,10}^{4}=P 3 g d r, b_{20,11}^{4}=$ $P 3 s d r, b_{21,12}^{4}=P 3 r d r, b_{28,25}^{4}=P 3 e d r, R B C t f_{13,1}=1, P L A T E L E T t f_{19,1}=1$. The other matrix elements are equal to zero.

\section{Further development}

To implement the effect of pro-drug Gangcyclovir (img) the elements of matrix $A$ are modified as follows:

$a_{1,1}(k)=\left(1+m_{s g}-a_{s g}-S g d r-C S_{g s}-C S_{g r}-C_{m y l} \times M y l_{s g} \times d_{m y l}(k) \quad-C_{g} \times\right.$ imgsens $\left._{s g} \times d_{\text {img }}(k)\right), \quad a_{2,2}(k)=\left(1+m_{S s}-a_{S s}{ }^{g s} S s d r-C S_{s g}^{g r}-C S_{s r}-C_{m y l} \times M y l_{S s} \times d_{m y l}(k)\right.$ $-C_{g} \times$ imgsens $\left._{S s} \times d_{i m g}(k)\right), \quad a_{3,3}(k)=\left(1+m_{S r}-a_{S r}-S r d r-\quad C S_{r s}-C S_{r g}-\right.$ $\left.C_{m y l}^{g} \times M y l_{S r} \times d_{m y l}(k) \quad-C_{g} \times \operatorname{imgsens}_{S r} \times d_{i m g}(k)\right), \quad a_{4,4}(k)=\left(1+m_{P I g}-a_{P 1 g}^{-}\right.$ $\left.P 1 g d r \quad-C P 1_{g s}-C P 1_{g r}-C_{m y} \times M y l_{P 1 g} \times d_{m y l}(k) \quad-C_{g} \times \operatorname{imgsens}_{P 1 g} \times d_{i m g}(k)\right)$, $a_{5,5}(k)=\left(1+m_{P 1 s}-a_{P 1 s}-P 1 s d r-C P 1_{s g}-C P 1_{s r}-C_{m y l}^{\delta} \times M y l_{P 1 s} \times d_{m y l}(k)\right.$ $-C_{g} \times$ imgsens $\left._{P 1 s} \times d_{i m g}(k)\right), \quad a_{6,6}(k)=\left(1+m_{P 1 r}-a_{P 1 r}-P 1 r d r-C P 1_{r g} \quad-C P 1_{r s}-\right.$ $C_{m y l}^{g} \times M y l_{P 1 r} \times d_{m y l}(k)-C_{g} \times$ imgsens $\left._{P 1 r} \times d_{i m g}(k)\right), a_{7,7}(k)=\left(1+m_{P 2 g}-a_{P 2 g}-P 2 g d r-\right.$ $C P 2_{g s}-C P 2_{g r}-C_{m y l} \times M y l_{P 2 g} \times d_{m y l}(k)-C_{g} \times$ imgsens $_{P 2 g} \times d_{i m g}(k)+C_{C Y T} \times d_{C Y T}(k)$ $\left.\times M_{C Y T}^{g s}\right), \quad a_{8, s}^{g r}(k)=\left(1+m_{P 2 s}-a_{P 2 s}-P 2 s d r-C P 2_{s g}-C P 2_{s r}-C_{m y l} \times M y l_{P 2 s} \times d_{m y l}(k)-\right.$ $C_{g} \times \quad$ imgsens $\left._{P 2 s} \times d_{i m g}(k)\right), \quad a_{9,9}(k)=\left(1+m_{P 2 r}-a_{P 2 r}-P 2 r d r-C P 2_{r g}-C P 2_{r s}-\right.$ $\left.C_{m y l}^{g} \times M y l_{P 2 r} \times d_{m y l}(k)-C_{g} \times i_{i m g s e n s} s_{P 2 r} \times d_{i m g}(k)\right), \quad a_{10,10}(k)=\left(1+m_{P 3 g}-a_{P 3 g}\right.$ $P 3 g d r-C P 3_{g s}-C P 3_{g r}-C_{m v l}^{g} \times M y l_{P 3 g} \times d m y l(k) \quad-C_{g} \times$ imgsens $\left._{P 3 g} \times d_{i m g}(k)\right)$, $a_{11,11}(k)=\left(1+m_{P 3 s}-a_{P 3 s}-P 3 s d r-C P 3_{s g}-C P 3_{s r}-C_{m y l} \times \quad M y l_{P 3 s} \times d m y l(k)-\right.$ $C_{g} \times$ imgsens $\left._{P 3 s} \times d_{i m g}(k)\right), \quad a_{12,12}(k)=\left(\begin{array}{llll}1 & +m_{P 3 r}-a_{P 3 r}-P 3 r d r & -C P 3_{r g} & -C P 3_{r s}-\end{array}\right.$ $C_{m y l}^{g} \times M y l_{P 3 r} \times d_{m y l}(k) \quad-C_{g} \times$ imgsens $\left._{P 3 r} \times d_{i m g}(k)\right), \quad a_{13,13}(k)=\left(1-a_{B 1 g}-C B 1_{g s}{ }^{-}\right.$ $C B 1_{g r}-C_{m y l} \times M y l_{B 1 g} \times d_{m y l}(k) \quad-C_{g} \times$ imgsens $\left._{B 1 g} \times d_{i m g}(k)\right), \quad a_{14,14}(k)=\left(1-a_{B 1 s}-\right.$ $\left.C B 1_{s g}{ }^{g}-C B 1_{s r}-C_{m y l} \times \quad M y l_{B 1 s} \times d_{m y l}^{g}(k)-C_{g} \times \operatorname{imgsens}_{B 1 s} \times d_{i m g}(k)\right), \quad a_{15,15}(k)=$ $\left(1-a_{B 1 r}-C B 1_{r g}{ }^{-} \quad C B 1_{r s}-C_{m y l} \times M y l_{B 1 r} \times d_{m y l}(k)-\quad C_{g} \times\right.$ imgsens $\left._{B 1 r} \times d_{i m g}(k)\right)$, $a_{16,16}(k)=\left(1-a_{B 2 g}-C B 2_{g s}-C B 2_{g r}-C_{m y l} \times M y l_{B 2 g} \times d_{m y l}^{g}(k)-C_{g} \times i_{i m g s e n s} \times\right.$ $\left.d_{i m g}(k)\right), \quad a_{17,17}(k)=\left(1-a_{B 2 s}-C B 2_{s g}-C B 2_{s r}-C_{m y l} \times M y l_{B 2 s} \times d_{m y l}^{g}(k) \quad-C_{g} \times\right.$ imgsens $\left._{B 2 s} \times d_{i m g}(k)\right), \quad a_{18,18}(k)=\left(1-a_{B 2 r}-C B 2_{r g}{ }^{i m}-C B 2_{r s}-C_{m y l} \times M y l_{B 2 r} \times d_{m y l}(k)-\right.$ $C_{g} \times$ imgsens $\left._{B 2 r} \times d_{i m g}(k)\right), \quad \quad a_{19,19}(k)=\left(1-a_{B 3 g}-C B 3_{g s}-C B 3_{g r}\right.$ $\left.C_{m y l}^{g} \times M y l_{B 3 g} \times d_{m y l}(k)-C_{g} \times i_{i m g s e n s} \times d_{B 3 g}(k)\right), \quad a_{20,20}(k)=\left(1-a_{B 3 s}-C B 3_{s g}{ }^{2}-\right.$ $C B 3_{s r}-C_{m y l} \times M y l_{B 3 s} \times d_{m y l}^{g}(k) \quad-C_{g} \times$ imgsens $\left._{B 3 s} \times d_{\text {img }}(k)\right), \quad a_{21,21}(k)=(1$ $-a_{B 3 r}-C B 3_{r g}{ }_{r g}-C B 3_{r s}{ }^{3}-C_{m y l} \times M y l_{B 3 r} \times d_{m y l}(k) \quad-C_{g} \times$ imgsens $\left._{B 3 r} \times d_{i m g}(k)\right)$, $a_{22,22}(k)=\left(1+m_{S e}-a_{S e}{ }^{-} \operatorname{Sedr}-\mathrm{C}_{\mathrm{im}} \times\right.$ imsens $\left._{S e} \times d_{i m}(k)\right), \quad a_{23,23}(k)=\left(1+m_{P 1 e}-\right.$ $\left.a_{P 1 e}-P 1 e d r-C_{g} \times i m g s e n s_{P 1 e} \times d_{i m g}(k)\right), \quad a_{24,24}(k)=\left(1+m_{P 2 e}-a_{P 2 e}-P 2 e d r\right.$ $-C_{g} \times$ imgsens $\left._{P 2 e} \times d_{i m g}(k)+C_{C Y T} \times d_{C Y T}(k) \times m_{D C Y T}\right), a_{25,25}(k)=\left(1+m_{P 3 e}-a_{P 3 e}-\right.$ P3edr $-C_{g} \times$ imgsens $\left._{P 3 e} \times d_{\text {img }}(k)\right), a_{26,26}(k)=\left(1-a_{B 1 e}-C_{g} \times\right.$ imgsens $\left._{B 1 e} \times d_{i m g}(k)\right)$, $a_{27,27}(k)=\left(1-a_{B 2 e}-C_{g} \times\right.$ imgsens $\left._{B 2 e} \times d_{\text {img }}(k)\right), \quad a_{28,28}(k)=\left(1-a_{B 3 e}-\right.$ $C_{g} \times$ imgsens $\left._{B 3 e} \times d_{i m g}(k)\right)$. The other elements of all the matrixes remain unchanged.

\section{Result}

For initial parametric values we have followed [16]. Free growth condition is depicted in (Figure 1 and Figure 2). Simulation observations suggest that with the increase in malignant cell population there is gradual decrease in the normal cell types in all the lineages. Conventionally in clinical practice HSCT is carried out after myeloablative chemotherapy and followed by different supportive therapy (i.e., transfusion of RBC and/or platelet). Here simulation is also carried out with this scheme. Simulation is carried out considering a worst leukemic condition. The differentiation rate of sensitive cell types is same as of resistive cell type in all the lineages and one-third cells become successful to be matured from progenitor cells.

Previously it has been shown that the day of transplantation is very crucial for successful outcome of stem cell transplantation. As per previous report [16] here, HSCT has been also carried out on day 180. Here simulation has been carried out with 100\% HLA mismatch (high GVHD). Under this condition it is observed that mature lymphocytes from the transplanted stem cells becomes operative (i.e., lymphocytes of donor origin are developed and starts killing of leukemic cells) after day $\sim 201$ and leukemic stem cells are removed from system on day $\sim 217$.

The application of doses of pro-drug in two different schemes are considered as 0.01 and 0.02 and drug sensitivities of different cell types are imgsens $_{S e}=$ imgsens $_{P 1 e}=$ imgsens $_{P 2 e}=$ imgsens $_{P 3 e}=$ imgsens $_{B 1 e}=$ imgsens $_{B 2 e}=$ imgsens $_{B 3 e}=0.92$ both for the first and the second doses. While the sensitivities of other cell types are assumed to be zero. The application of pro-drug Gangcyclovir is applied on day 210 with a low dose; however, application of high dose is started on day 240 along the continuation of low dose (Figure 3 ). Our simulation results suggest that if the low dose application is absent or only low dose is (a) Free growth

(i) Stem Cell

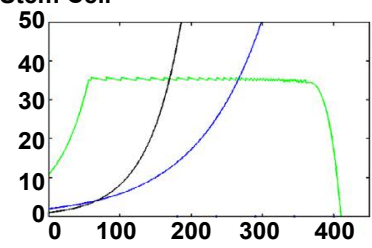

(ii) Erythroblast

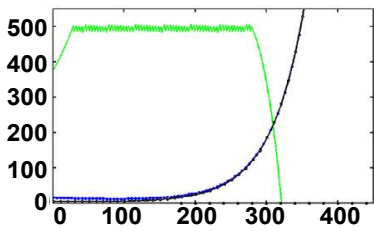

(iii) Leukoblast

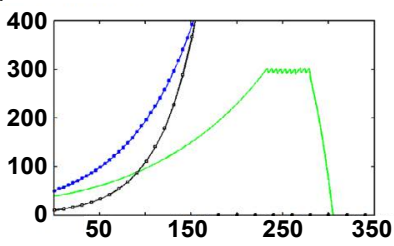

(iv) Megakaryoblast

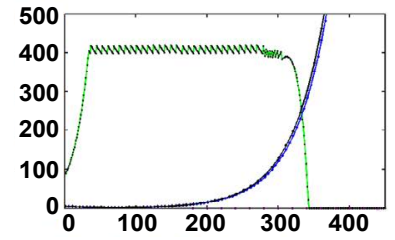

(b) MYL followed by Gene therapy (i) Stem Cell

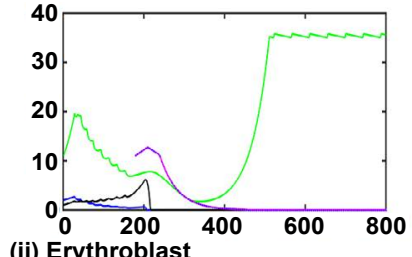

(ii) Erythroblast

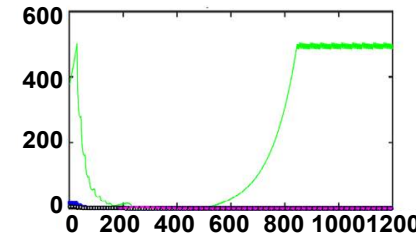

(iii) Leukoblast

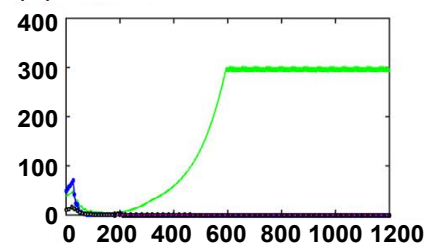

(iv) Megakaryoblast

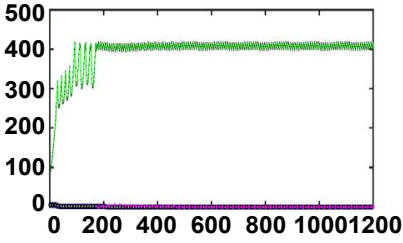

Figure 1: Removal of leukemia after conventional myeloablative chemotherapy followed by stem cell transplantation with suicidal gene construct. Plots showing the behavior of stem cells (i), erythroblasts (ii), leukoblasts (iii), megakaryoblasts (iv) under freely growing condition (a) and with conventional chemotherapy followed by HSCT with tk-gene construct therapy (b). In all the plots, $x$-axis represents 'Days' and $y$-axis represents 'Counts'. In all the plots, green, blue, black and magenta lines represent nonmalignant, sensitive type malignant, resistive type malignant cell populations and cells with donor origin respectively. 
(a) Free growth

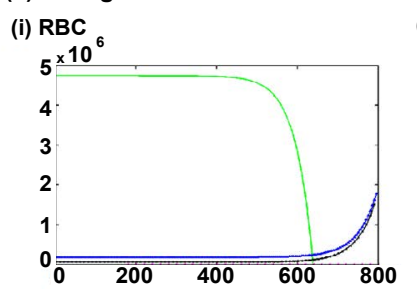

(ii) WBC

(iii) Platelet

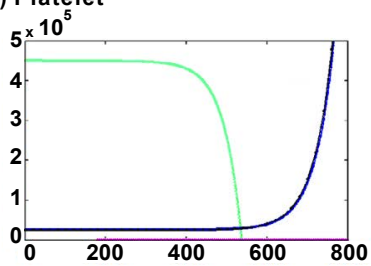

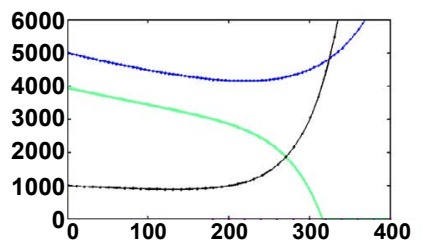

(b) MYL followed by Gene therapy (i) RBC

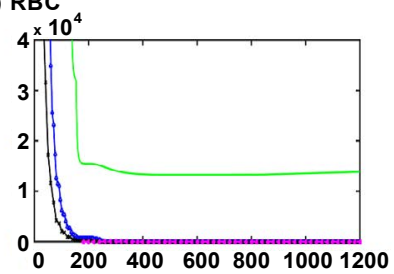
(ii) WBC

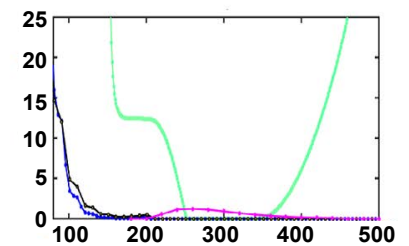

(iii) Platelet

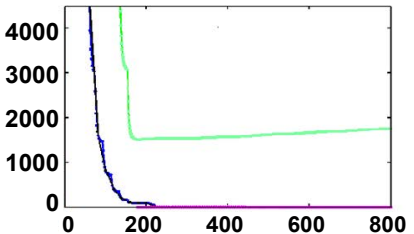

Figure 2: Removal of leukemia after conventional myeloablative chemotherapy followed by stem cell transplantation with suicidal gene construct. Plots showing the behavior of RBCs (i), WBCs (ii), platelets (iii) under freely growing condition (a) and with conventional chemotherapy followed by HSCT with tkgene construct therapy (b). In all the plots, $x$-axis represents 'Days' and y-axis represents 'Counts'. In all the plots, green, blue, black and magenta lines represent nonmalignant, sensitive type malignant, resistive type malignant cell populations and cells with donor origin respectively.

continued then there is chance of GVHD related mortality. Contrarily if high dose is applied early then there is chance of residual leukemia due to suppression of development of lymphocytes of donor origin. So, optimization in the application of drug schedule and drug dose is very important in the successful outcome of HSCT with $t k$-gene construct.

This therapeutic model is developed from the earlier three compartmental hematopoiesis model where methodology of incorporation of stochastic process at the parametric level has already been shown [19]. To keep the simulation study simple we have avoided this in the present simulation runs. However one can incorporate the necessary stochastic components to test the effect of stochastic behaviors of individual system parameters towards the cancer dynamics as the developed model is flexible in nature.

\section{Discussion}

Simulation runs showed that with allo-HSC transplantation with high HLA mismatch may be helpful in removal of malignancy but it requires a minimum kill factor (minimum killing efficiency of the developed lymphocytes from transplanted cells of donor origin). Though with the high HLA mis-match between donor and recipient there is possibility of high GVHD and GVHD related death of the recipient, but transplanted stem cells having the suicidal $(t k)$ gene construct provides a control measure to GVHD mortality. Our study indicates that this scheme provides the opportunity of successful outcome of HSCT without any GVHD related detrimental effect on recipient. However, our model and simulation study also indicate that optimization of drug schedule (drug dose and time of drug application) is a crucial factor for the successful outcome of this therapy. Since earlier application of higher dose may stop the development of the donor lymphocyte thereby restrict the removal of malignant cell while after malignancy removal if lymphocytes donor origin are not removed from the host system then it may cause GVHD related mortality. Our model also indicates that the threshold of drug sensitivity of the lymphocytes is another important criteria and needed to check before going to such therapy and depending upon this factor, drug dose and drug application time are needed to be adjusted further.

Recent time for leukemia therapy, HSCT with suicidal $t k$ gene construct is suggested by the different experts of the concerned scientific community $[17,18]$. However, due to unavailability of suitable analytical method, this procedure may be far from clinical practice. Here it is shown that the previously developed model [16] for leukemia therapy can be fitted for the assessment of such therapeutic procedure with a minor modification of variables and thus, provides the desired platform to bring such excellent therapeutic procedure in reality. Presently, systems biologists are dependent on reverse engineering and from this stand-point, simulation runs with fitting of different parametric values of different systems component to an analytical model can help to identify the controlling variable(s) and their extent (boundary conditions) as well as its impact on the systems dynamical behavior. After applying any newer treatment procedure for a shorter duration of time, reassessment of the variables is needed and if there is any deviation between the simulation output and real life situation, then readjustment of initial parametric values of different variables are needed. Hence it will follow the predict-observe-correct cycle $[16,20,21]$. It is needless to point out here that as the developed model is based on difference equation and different feedbacks are incorporated in the form of inequation, so the model has the flexibility to change its parametric values of different variables and hence investigator can change the parametric values of any variables. Moreover, all the considered variables like cell counts, multiplication rate, differentiation time and leukemic cell killing capacity of immunocytes or drug can be captured by different hematological, immunological and cell culture based investigations.

Though HSCT with $t k$-gene construct has an immense potentiality for leukemia treatment; however, still is in laboratory phase. Presently cancers including leukemia treatment of individual patients are
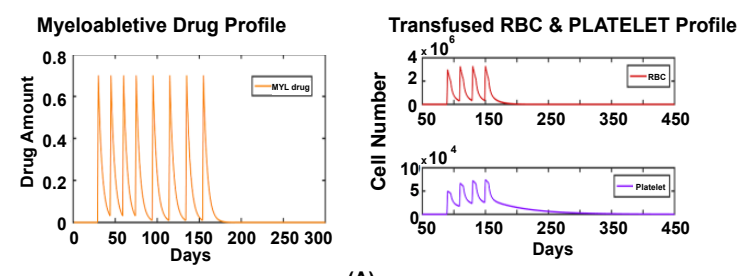

(A)
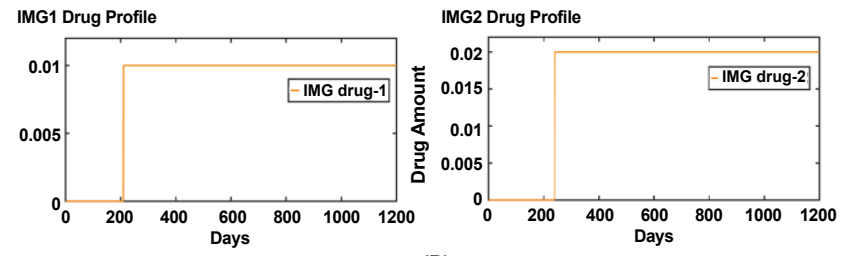

(B)

Figure 3: In (A), plots showing MYL drug profile, transfused RBC and platelets profile; in (B), plots showing Pro-drug with lower dose (IMG-1) and higher dose (IMG-2) profile and drug doses are represented as \% of leukemic cell killing per day. 
Citation: Dhar PK, Naskar TK, Majumder D (2015) Analytical Model for the Assessment of Efficiency of Stem Cell Transplantation with Suicidal Gene Construct for the Treatment of Leukemia. J Oncol Transl Res 1: 103. 10.4172/2476-2261.1000103

Page 5 of 5

dependent on population based clinical trials. Recent time emphasis is imparted towards individualized treatment procedure. Though several large databases on leukemia are available; however, treatment dynamical data in public domain are unavailable. Moreover, clinically relevant suitable analytical platform is also unavailable; hence, priority in the area of Cancer Systems Biology is given towards the development of analytical model with a strong clinical rationality; so that, in future the efficacy and outcome of any newer cancer treatment procedure can be tested apriori by fitting the individual patients' initial parametric values of different variables along with different therapeutic schedule of clinicians' choice $[16,20,22]$. Towards this goal, this analytical model is developed.

\section{Acknowledgment}

Authors acknowledge the critical comments of the eminent founder members of the Society for Systems Biology \& Translational Research.

\section{References}

1. Corsten MF, Shah K (2008) Therapeutic stem-cells for cancer treatment: hopes and hurdles in tactical warfare. Lancet Oncol 9: 376-384.

2. Szabolcs $P(2010)$ Selecting unrelated donor grafts to prevent leukaemia relapse.Lancet Oncol 11: 608-609.

3. Rubinow SI, Lebowitz JL (1976) A mathematical model of the acute myeloblasticleukemic state in man.Biophys $\mathrm{J}$ 16: 897-910.

4. Rubinow SI, Lebowitz JL (1976) A mathematical model of the chemotherapeutic treatment of acute myeloblastic leukemia. Biophys J 16: 1257-1271.

5. Djulbegovic B, Svetina S (1985) Mathematical model of acute myeloblasticleukaemia: an investigation of the relevant kinetic parameters. Cell Tissue Kinet 18: 307-319.

6. Colijn C, Mackey MC (2005) A mathematical model of hematopoiesis - I. Periodic chronic myelogenous leukemia. J Theor Biol 237: 117-132.

7. Colijn C, Mackey MC (2005) A mathematical model of hematopoiesis: II Cyclical neutropenia. J Theor Biol 237: 133-146.

8. Michor F, Hughes TP, Iwasa Y, Branford S, Shah NP, et al. (2005) Dynamics of chronic myeloid leukaemia. Nature 435: 1267-1270.

9. Kim PS, Lee PP, Levy D (2008) Dynamics and potential impact of the immune response to chronic myelogenous leukemia. PLoS Comput Biol 4: e1000095.
10. Peet MM, Kim PS, Niculescu S-I, Levy D (2009) New computational tools for modeling chronic myelogenous leukemia. Math Model Nat Phenom 4: 48-68.

11. Fokas AS, Keller JB, Clarkson BD (1991) Mathematical model of granulocytopoiesis and chronic myelogenous leukemia. Cancer Res 51: 20842091

12. Moore H, Li NK (2004) A mathematical model for chronic myelogenous leukemia (CML) and T cell interaction. J Theor Biol 227: 513-523.

13. Cucuianu A, Precup R (2010) A hypothetical-mathematical model of acute myeloid leukemia pathogenesis.Comput Math Methods Med 11: 49-65.

14. DeConde R, Kim PS, Levy D, Lee PP (2005) Post-transplantation dynamics of the immune response to chronic myelogenous leukemia. J Theor Biol 236 : $39-59$

15. Roeder I, Horn M, Glauche I, Hochhaus A, Mueller MC, et al. (2006) Dynamic modeling of imatinib treated chronic myelogenous leukemia: functional insights and clinical implication Nat Med 12: 1181-1184.

16. Dhar PK, Majumder D (2015) Development of the analytical model for the assessment of the efficiencies of different therapeutic modalities in leukaemia. J Comp Syst Biol 1: (Article ID 104) 1-45.

17. Zhan H, Gilmour K, Chan L, Farzaneh F, McNicol AM, et al. (2013) Production and first-in-man use of T cells engineered to express a HSVTK-CD34 sortsuicide gene. PLoS One 8: e77106.

18. Greco R, Oliveira G, Stanghellini MT, Vago L, Bondanza A, et al. (2015) Improving the safety of cell therapy with the TK-suicide gene. Front Pharmacol 6: 95

19. Dhar PK, Mukherjee A, Majumder D (2012) Difference delay equation based analytical model of hematopoiesis. Auto Contrl Physiol State Func 1: (Article ID 235488) 1-11.

20. Majumder D, Mukherjee A (2011) A passage through systems biology to systems medicine: adoption of middle-out rational approaches towards the understanding of therapeutic outcomes of cancer. Analyst 136: 663-678.

21. Dhar PK, Majumder D (2013) A composite synergistic systems model fo exploring the efficacies of different chemotherapeutic strategies in cancer. Comp Biol J 2013: (Article ID 301369) 1-26.

22. Majumder D, Mukherjee A (2013) Multi-scale modeling approaches in systems biology towards the assessment of cancer treatment dynamics: adoption of middle-out rationalist approach. Adv Cancer Res Treat 2013: (Article ID 587889) 1-26. 